Meta

Journal des traducteurs

Translators' Journal

\title{
Marketing, commercialisation, mercatique, marchéage, mise en marché
}

\section{Noëlle Guilloton}

Volume 22, numéro 3, septembre 1977

URI : https://id.erudit.org/iderudit/002986ar

DOI : https://doi.org/10.7202/002986ar

Aller au sommaire du numéro

Éditeur(s)

Les Presses de l'Université de Montréal

ISSN

0026-0452 (imprimé)

1492-1421 (numérique)

Découvrir la revue

Citer cet article

Guilloton, N. (1977). Marketing, commercialisation, mercatique, marchéage,

mise en marché. Meta, 22(3), 211-217. https://doi.org/10.7202/002986ar d'utilisation que vous pouvez consulter en ligne.

https://apropos.erudit.org/fr/usagers/politique-dutilisation/ 


\section{MARKETING, COMMERCIALISATION, MERCATIQUE, MARCHÉAGE, MISE EN MARCHÉ}

L'étude qui suit porte sur les cinq termes concurrents énumérés ci-dessus et se rapportant, semble-t-il, à une seule et même notion. Il s'agira donc de définir cette notion, puis de compiler les solutions proposées et de critiquer les termes retenus, pour en arriver au choix d'un terme.

Que recouvre la notion de «marketing» en anglais?

Le Random House Dictionary ${ }^{1}$ la définit ainsi :

The total of activities by which transfer of title or possession of goods from seller to buyer is effected, including advertising, shipping, storing, and selling. 
En français, il semble que la notion et le terme de «marketing » - tous deux d'origine anglo-saxonne - soient souvent indissociables, à en juger par la définition qu'en donne le Centre international du droit des affaires de Paris :

Accomplissement d'activités commerciales destinées à faire passer les biens et services du producteur au consommateur ou à l'utilisateur ${ }^{2}$.

ce qui est la traduction fidèle de l'American Marketing Association :

The performance of business activities that direct the flow of goods and services from producers to consumers or users ${ }^{3}$.

Cependant, le Dictionnaire de l'organisation et de la gestion est plus précis :

Méthode scientifique de détection et de conquête rentable des marchés par la recherche des stratégies commerciales les plus efficaces et le contrôle des opérations lancées en exécution de la stratégie choisie. [...] Etat d'esprit qui s'attache à l'idée de déceler les besoins et les désirs des utilisateurs ou consommateurs... Ce concept englobe la recherche et l'étude du marché... la promotion des ventes ${ }^{4}$.

et, dans le même esprit, Hure donne du marketing (angl.) la définition suivante :

Toutes techniques, études, recherches, prospections visant à saisir l'étendue et les caractéristiques du marché, méthodes et moyens de toucher et d'influencer les acheteurs éventuels, adaptation de la production aux besoins et toutes opérations de distribution et de commercialisation proprement dites ${ }^{5}$.

En passant, on peut remarquer le nombre de descripteurs communs à ces deux définitions, ce qui nous permet de retenir ces dernières sans grand risque d'erreur.

Le terme «marketing» n'est cependant pas seul en lice pour rendre la notion définie ci-dessus; à titre documentaire, voici une liste non exhaustive des solutions qui sont proposées dans diverses sources (les numéros accompagnant chaque terme renvoient aux notes données dans l'article) :

action commerciale : 18

commercialisation : $4,10,12,14,20,22,25$

commercialisation d'un produit (Etiemble)

conquête des marchés : 23

étude de marché

gestion commerciale : 18

étude de marché et action en retour : 18

marchéage

marketing : $1,2,6,7,8,9,14,15,16,19$

mercatique : 5

mercantique : 18

mercatonomie : 24

mise en marché

négoce : 18

négocialogie : 24

négociologie : 18

techniques commerciales : 15,22 
Rappelons toutefois que la suite de l'étude ne concernera que les cinq termes suggérés initialement : nous ferons une critique de chacun d'entre eux pour analyser le plus précisément possible leur formation, l'usage qui en est fait, et leur adéquation à la notion étudiée.

Comme nous l'avons vu plus haut, le terme «marketing» a été adopté dans les pays francophones en même temps que les activités et les techniques qu'il désigne. Son usage est très répandu dans les revues ${ }^{6}$ et ouvrages spécialisés ${ }^{7}$ et 8 et il y devance nettement ses quatre concurrents. Il faut dire que le grand nombre d'ouvrages traduits de l'américain - où tout est traduit, sauf le terme «marketing 》 lui-même - y est pour beaucoup dans la diffusion de ce terme, ces ouvrages étant sans doute antérieurs à l'application généralisée de la technique «marketing» au monde francophone des affaires. L'usage de «marketing » s'est étendu, des catalogues des bibliothèques (dans celui de la bibliothèque des H.E.C., la vedette-matière "marketing » est donnée à plus d'un millier de fiches) aux divers glossaires, lexiques, dictionnaires et thésauri $2,4,9,10,11,12,13,14$.

Le roi-usage nous ordonnerait d'arrêter là notre étude, d'autant plus que «marketing» rallie en sa faveur de nombreux avis autorisés : ceux de «professeurs de marketing », évidemment.

C'est ainsi qu'il n'était plus possible d'ignorer que le concept et l'esprit de marketing avaient fini par supplanter, dans le milieu des affaires, de l'enseignement et de la recherche, le concept des techniques commerciales. En rebaptisant l'ouvrage Encyclopédie du Marketing, le comité de rédaction a voulu, au-delà des querelles linguistiques, rendre compte d'une évolution des pensées maintenant irréversible. Le terme marketing recouvre une réalité actuelle de la gestion des entreprises : faire du marketing, c'est se préoccuper d'abord de connaître son marché, ses besoins, ses tendances, afin de mettre au point une stratégie d'attaque de ce marché, les techniques commerciales n'intervenant que comme outils de saisie du réel et d'élaboration de la stratégie ${ }^{15}$.

Et ceux d'auteurs de traités spécialisés :

Les amoureux de la langue française vont se récrier : il faut bien le reconnaître, on n'a pas encore trouvé de terme aussi concis et aussi précis en français pour exprimer des notions aussi complexes que celles de marketing et de merchandising ${ }^{18}$.

Mais oui, justement, les « amoureux de la langue française » formulent différentes objections à l'égard de ce nouvel emprunt, et ce serait faire injure à la langue française que de sous-estimer sa capacité à rendre compte de la complexité de cette notion.

Alfred Sauvy, professeur au Collège de France et membre du Conseil économique et social de ce pays, écrivait déjà, en 1964 : «Si l'on veut bien fermer les oreilles devant l'affreux "marketing », on ne peut qu'être confondu devant l'ignorance qui a si longtemps prévalu sur la connaissance des marchés ${ }^{17}$. " (Pensait-il donc que la technique - souhaitable - et son appellation - affreuse - ne peuvent pas être dissociées?) 
Le premier inconvénient de «marketing», c'est donc sa sonorité absolument étrangère aux « oreilles » françaises :

J'ajoute que l'oreille me blesse d'une autre manière, et au sens propre, car si chacun parle de marketing, la prononciation en est faite tantôt à l'américaine, tantôt, et le plus souvent, d'une façon intermédiaire entre l'anglais et le français. Si l'on voulait transposer littéralement le terme en français, en adoptant seulement l'orthographe, comme on le fait parfois (accords de soipe...) il faudrait choisir avec celle-ci une prononciation convenable. Détail, mais raison de plus pour vraiment traduire ${ }^{18}$.

De plus, (un malheur ne venant jamais seul...), l'adoption de * marketing * doit nécessairement être suivie, en toute logique, d'une série d'autres emprunts comme : « marketing-mix », « gestion marketing » ou «management marketing 》, etc., et cette prolifération de termes coïncidant avec les développements de la technique serait inquiétante. Des auteurs vont même jusqu'à composer, au gré de leur fantaisie, des mots avec "marketing», ce qui confère à ceux-ci des accents quelque peu «bâtards »: «comportement marketing ${ }^{3}$, «stratégie marketing ${ }^{7} \gg$, etc., à moins que marketing soit ici adjectif ?

Pourquoi donc ne pas se tourner plutôt vers d'autres solutions, néologismes et autres?

Le terme «commercialisation» date de 1922 : ce n'est donc plus un néologisme, et le Robert en donne une définition des plus laconiques : « Action de commercialiser» (commercialiser signifiant : rendre quelque chose l'objet d'un commerce). Y a-t-il adéquation entre ce terme et la notion cernée plus haut? Si la définition du Robert ne nous éclaire guère sur ce point, par contre, une étude de M. Marcel Lagrenade, alors directeur du Service de traduction à la Domtar, nous indique que non, la commercialisation étant d'après lui la partie $\mathrm{du}$ marketing qui commence avec le conditionnement du produit et se termine avec le service après-vente, et n'englobant ni les études préliminaires et la fabrication, ni les études terminales ${ }^{19}$. Guillaumont est également de cet avis : pour lui «si les mots dérivés de «commerce» ne conviennent pas, ce n'est pas parce que le commerce n'est pas honorable, c'est parce qu'il n'exprime qu'en partie la relation entre le marché et la production ${ }^{18}$.

De plus, l'O.C.D.E. ${ }^{14}$ emploie et marketing et commercialisation : ne peut-on en déduire qu'il s'agit bien là de deux concepts différents? Notons également que le Dictionnaire des sciences de la gestion ${ }^{10}$, qui a une vedette «marketing \$ en a aussi une «commercialisation » dont la définition est : "mise du produit sur le marché ».

Il n'en reste pas moins que ce terme trouve, pour cette acception, de nombreux défenseurs, et non des moindres. L'Académie française le propose comme traduction de «marketing», ainsi que Dubuc et bien d'autres, dont Lauzel qui en donne la définition suivante, assurément des plus larges :

La commercialisation est l'ensemble des activités qui interviennent depuis et y compris la conception d'un produit ou d'un service jusqu'à sa destruction par le consommateur final ${ }^{11}$. 
Il est également proposé comme remplaçant facultatif de marketing - et mis sur le même pied que ce dernier terme - dans plusieurs sources, et en particulier dans le cahier $\mathrm{n}^{\circ} 24$ de l'Office de la langue française : "Vice-président à la commercialisation ou Vice-président au marketing ${ }^{20} \gg$.

Par contre, à la quatrième Biennale de la langue française (1974), ce n'est pas le terme «commercialisation» qui fut retenu, mais l'expression voisine «techniques commerciales», avec $50 \%$ des suffrages.

Nous pouvons donc conclure qu'il n'y a pas consensus au sujet de «commercialisation » et, ce qui est plus grave, qu'il y a même risque de collision sémantique, le terme étant pour certains «tout », pour d'autres «partie ».

Le néologisme «mercatique » a pour racine le latin mercator (marchand) auquel on a adjoint le suffixe latin ou grec -ique (les deux sont confondus, d'après Grevisse) qui appartient à la terminologie scientifique. Assez savant donc, mais de dérivation facile, «mercatique » peut aussi être adjectif et faciliter la formation d'expressions : «gestion mercatique », «stratégie mercatique », «comportement mercatique », etc., toutes des plus acceptables.

Dès 1971, Hure le propose comme équivalent français de marketing (angl.), et en donne la définition citée plus haut.

L'année suivante, «mercantique », terme jumeau (la seule différence entre ces deux termes est que mercantique est plus proche des mots péjoratifs mercantile et mercanti) est proposé par Guillaumont et fort bien défendu par lui :

Recourant à la néologie, je proposerais pour ma part «mercantique » (substantif féminin : la mercantique). Le terme évoque le marketing, avec une énergie voisine, une référence au marché, mais avec une consonance nettement française, disons latine... Les dérivés suivent facilement : gestion ou ménagement mercantique (marketing management)... stratégie mercantique (marketing mix), mercantistes (spécialistes de mercantique); de même les applications : mercantique industrielle, mercantique agricole... Certes le mot n'est pas sans défaut. Mercantique : ne voit-on pas déjà se faufiler les 《mercantis », se préparer des actions «mercantiles » et ressurgir à la suite le préjugé relatif au commerce... En fait la qualification des mots, leur caractère éventuellement péjoratif leur vient plus souvent de leur terminaison que de leurs racines : tout assoiffé n'est pas un soiffard... En l'occurrence, les économistes et les historiens de la pensée sont habitués à parler de mercantilisme, sans aucune nuance péjorative. D'une part, il y a quelque hypocrisie sociale à ne pas désigner les choses par leur nom, ici à recourir à un terme anglais qui suggère la technique et le modernisme, mais qui d'abord se rapporte à une action effectuée à partir du marché ${ }^{18}$.

Bien que d'usage encore rare, "mercatique» est déjà employé par des organismes qui, comme Informatech France-Québec, tiennent à leur image francophone.

Le néologisme «marchéage » quant à lui, est très motivé des points de vue étymologique et morphologique :

marché + age (suffixe populaire) 
il fait partic des onze termes proposés aux participants à la quatrième Biennale de la langue française, mais n'y a guère eu de succès. Craindrait-on, par association phonique avec marchandage, une connotation péjorative? Quoi qu'il en soit, son usage est extrêmement rare.

Pour terminer cette revue, il faut admettre que l'expression «mise en marché » n'a pas toute l'extension de "marketing et ne s'applique, d'après Lagrenade ${ }^{19}$, qu'aux étapes allant de l'étalage à la vente du produit. Serait-ce alors plutôt un équivalent de marchandisage (merchandising) ? ting ${ }^{21} \gg$.

Toutefois, Lebel donne cette expression comme traduction de «marke-

De toute façon, il est toujours préférable de manier un mot seul, ce qui facilite souvent la dérivation.

Nous pouvons maintenant nous pencher sur un tableau comparatif des cinq termes étudiés, dans lequel ceux-ci sont évalués en fonction de certains critères propres à la normalisation. (La pondération, qui ne peut être que subjective, est établie de 1 à 10.)

\begin{tabular}{|c|c|c|c|c|c|c|c|}
\hline & 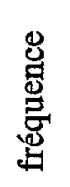 & 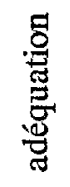 & 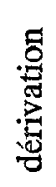 & 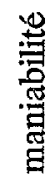 & 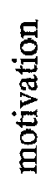 & 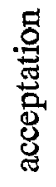 & 芯 \\
\hline Marketing & 10 & 9 & 0 & 2 & 0 & 2 & 23 \\
\hline Commercialisation & 4 & 5 & 7 & 7 & 8 & 7 & $38 / 34$ \\
\hline Mercatique & 2 & 8 & 8 & 8 & 6 & 7 & $39 / 37$ \\
\hline Marchéage & 1 & 6 & 5 & 7 & 6 & 2 & 27 \\
\hline Mise en marché & 2 & 2 & 0 & 2 & 8 & 7 & 21 \\
\hline
\end{tabular}

D'après ce tableau, "commercialisation » et «mercatique » sont presque ex aequo, mais si l'on exclut le critère de la fréquence - qui ne contribue en l'occurrence qu'à favoriser l'emprunt et qui perd de sa valeur lorsqu'il s'agit de néologismes -, la différence augmente quelque peu à l'avantage de «mercatique $»$.

Alors, même si le «marketing » n'est pas encore mort, vive la «mercatique $\gg !$

NoËLle GUILLOTON 


\section{NOTES}

1. The Random House Dictionary of the English Language, The Unabridged Edition, New York, Random House, c1973, 2059 p.

2. Centre international du droit des affaires, Lexique pratique commercial, Paris, REGIF, 1973, $451 \mathrm{p}$

3. American Marketing Association, Committee on Definitions, Marketing Definitions : A Glossary of Marketing Terms, Chicago, American Marketing Association, 1960, p. 15.

4. J. Tezenas, Dictionnaire de lorganisation et de la gestion, Paris, Les éditions d'organisation, $1971,270 \mathrm{p}$.

5. Joseph Hure, «La terminologie pétrolière $\triangleright$, la Banque des mots, no 2, 1971, p. 191.

6. Revue française du marketing, Paris, Association nationale pour le développement des techniques de marketing, 1964.

7. Michel Chevalier, la Stratégie marketing: le plan de marketing et la stratégie commerciale, synthèse et recueil de textes classiques, Paris, Presses Universitaires de France, 1975, $283 \mathrm{p}$

8. Bernard Yon, le Comportement marketing de l'entreprise, une approche économétrique Paris, Dunod, 1976. (Coll. Dunod entreprise, Gestion commerciale et marketing), 115 p

9. François Roche, Lexique du marketing, $3^{\mathrm{e}}$ édition, Paris, Entreprise moderne d'édition, $1970,108 \mathrm{p}$.

10. Henri Tézenas du Montcel, Dictionnaire des sciences de la gestion, Paris, Mame, 1972, $331 \mathrm{p}$.

11. Pierre Lauzel, Lexique de la gestion, Paris, Entreprise moderne d'édition, 1970, 237 p.

12. Georges Anderla, Dictionnaire des affaires, Paris, Delmas, $1972,587 \mathrm{p}$.

13. Henri Van Hoof, Terminologie économique, Paris, Dunod, c1967, $770 \mathrm{p}$.

14. Organisation de coopération et de développement économique, Liste commune des descripteurs : développement économique et social, Paris, O.C.D.E., 1969.

15. Techniques commerciales-encyclopédie du marketing, collection dirigée par Michel Chevalier et al., Paris, Editions techniques, 1975, vol. 1, p. 3.

16. Jean Saint-Cricg et Olivier Bruel, la Pratique du merchandising, Paris, Les éditions d'organisation, 1973, $292 \mathrm{p}$.

17. Alfred Sauvy, « Préface s, Revue française de marketing, no 10,1964, p. 4.

18. P. Guillaumont, "Marketing ou mercantique? ", Annales économiques, $\mathrm{n}^{\mathrm{o}}$ 3, 1972 , p. 257-259.

19. Québec (Prov.), Office de la langue française, la Normalisation linguistique; actes du colloque international de terminologie, Québec, 1974, p. 228 (annexe IV).

20. Québec (Prov), Office de la langue française, Terminologie de la gestion : les organigrammes, Cahier de l'Office de la langue française, $\mathrm{n}^{\circ} 24, \mathrm{p} .83$.

21. Wilfrid Lebel, le Dictionnaire des affaires, Montréal, Editions de l'homme, c1967, 80, $78 \mathrm{p}$.

22. Robert Dubuc Vocabulaire de gestion, Montréal, Leméac, c1974, 135 p.

23. F. Nepveu-Nivelle, Conquête des marchés ou le marketing à l'européenne, Paris, Dunod, 1959, 197 p.

24. Denis Miannay, "Les auditeurs de l'ORTF et la création lexicale ", la Banque des mots, $\mathrm{n}^{\circ} 3,1972$, p. 11 .

25. Robert Leduc, Comment lancer un produit nouveau; conception, développement, commercialisation, Paris, Dunod, 1972, 217 p. 